\title{
Bristow artroscópico: Avaliação da segurança e da eficácia, 12 anos de experiência*
}

\section{Arthroscopic Bristow: Assessments of Safety and Effectiveness, 12 Years of Experience}

Jose Carlos Garcia Júnior ${ }^{10}$

\author{
${ }^{1}$ Grupo de Ombro, Núcleo Avançado de Estudos em Ortopedia e \\ Neurocirurgia (Naeon), São Paulo, São Paulo, Brasil
}

Rev Bras Ortop 2021;56(2):205-212.

\begin{abstract}
Endereço para correspondência Jose Carlos Garcia Júnior, PhD, Núcleo Avançado de Estudos em Ortopedia e Neurocirurgia, Avenida Ibiraquera 2.144, Moema, São Paulo, SP, 04028-001, Brasil (e-mail: josecarlos@naeon.org.br).
\end{abstract}

\section{Resumo}

Palavras-chave

- procedimentos ortopédicos

- instabilidade do ombro

- luxação do ombro

- artroscopia
Objetivo O procedimento de Bristow aberto é um método há muito estabelecido e eficaz no tratamento da instabilidade anterior do ombro. Seguindo as tendências das cirurgias minimamente invasivas, essa cirurgia foi realizada por artroscopia, e seus resultados foram avaliados.

Métodos Foram avaliados 43 ombros de pacientes submetidos ao procedimento de Bristow por artroscopia, com o enxerto em posição horizontal e uso de um parafuso, com pelo menos dois anos de seguimento pós-cirúrgico, por meio de escores qualidade de vida, índice de reluxação e perda de rotação lateral.

Resultados A média de seguimento foi de 76 meses (variando de 129 a 24 meses), e o escore da University of California at Los Angeles (UCLA) variou de 25,56 $\pm 0,50$ (desvio padrão $[D P]=3,25)$ para 33,23 $\pm 0,44(D P=2,91)(p<0,0001)$. A média para o escore de Rowe com 2 anos ou mais de cirurgia foi de $94,25 \pm 1,52(D P=1,34$ ), sendo que $o$ padrão de bons resultados é de 75 pontos $(p<0,0001)$. A média do teste simples de ombro foi de $11,35 \pm 0,21$ ( $D P=1,34)$, e, para perda de rotação lateral, foi de $10,37^{\circ} \pm 1,36^{\circ}\left(\mathrm{DP}=8,58^{\circ}\right)$. Não houve reluxações.

Entre os 43 pacientes operados, ocorreram um total de 12 complicações, das quais 8 não apresentaram qualquer repercussão clínica. As complicações com repercussão clínica foram uma infecção de possível origem hematogênica seis meses após a cirurgia, uma fratura do coracoide que fez com que o paciente precisasse mudar o procedimento no intraoperatório, e dois pacientes com impacto anterior, que necessitaram de retirada de material de síntese mais de seis meses após a cirurgia.

Conclusão O procedimento de Bristow artroscópico mostrou eficácia no tratamento da instabilidade anterior do ombro, embora não seja livre de complicações.
Trabalho feito no Grupo de Ombro, Núcleo Avançado de Estudos em Ortopedia e Neurocirurgia (Naeon), São Paulo, São Paulo, Brasil.

\section{recebido}

21 de Janeiro de 2019

aceito

18 de Julho de 2019

Publicado online

Dezembro 13, 2019
DOI https://doi.org/

10.1055/s-0039-1697972. ISSN 0102-3616.
(C) 2019. Sociedade Brasileira de Ortopedia e Traumatologia. All rights reserved.

This is an open access article published by Thieme under the terms of the Creative Commons Attribution-NonDerivative-NonCommercial-License, permitting copying and reproduction so long as the original work is given appropriate credit. Contents may not be used for commercial purposes, or adapted, remixed, transformed or built upon. (https://creativecommons.org/ licenses/by-nc-nd/4.0/)

Thieme Revinter Publicações Ltda., Rua do Matoso 170, Rio de Janeiro, RJ, CEP 20270-135, Brazil 


\begin{abstract}
Keywords

- orthopedic procedures

- shoulder instability

- shoulder dislocation

- arthroscopy

Objective The open Bristow procedure is a long established and effective method for treating anterior shoulder instability. Following the trends of minimally-invasive surgeries, these procedures were performed arthroscopically, and their outcomes were evaluated.

Methods A total of 43 shoulders of patients submitted to Bristow procedures by arthroscopy, using a graft positioned horizontally and a screw, with at least two years of postoperative follow-up, were evaluated regarding quality of life, de novo dislocation index, and loss of lateral rotation.

Results The mean follow-up time was of 76 months (range: 129 to 24 months). The University of California at Los Angeles (UCLA) score varied from $25.56 \pm 0.50$ (standard deviation $[S D]=3.25)$ to $33.23 \pm 0.44(S D=2.91)(p<0.0001)$. Two or more years after surgery, the mean Rowe score was of $94.25 \pm 1.52(S D=1.34)$, whereas the good results standard is $75(p<0.0001)$. The mean value for the simple shoulder test was of $11.35 \pm 0.21(S D=1.34)$, while the mean value of the lateral rotation loss was of $10.37^{\circ} \pm 1.36^{\circ}\left(\mathrm{SD}=8.58^{\circ}\right)$. There were no de novo dislocations.

In total, there were 12 complications, 8 of which had no clinical repercussions. The clinically-significant complications included an infection six months after surgery with a potential hematogenous origin, a coracoid fracture that required an intraoperatively procedure change, and two patients with previous impingement who required synthesis material removal more than six months after surgery.

Conclusion Although the arthroscopic Bristow procedure was effective in treating anterior shoulder instability, it is not a complication-free surgery.
\end{abstract}

\section{Introdução}

A instabilidade anterior é uma das condições ortopédicas mais comuns na cirurgia do ombro. Nos Estados Unidos, por exemplo, ela atinge taxas de até 23,9 casos por 100.000 pessoas por ano. ${ }^{1}$

Entre as técnicas cirúrgicas para se tratar essa condição, uma das mais eficazes e conhecidas é a transferência do processo coracoide e do tendão conjunto para a borda anterior da glenoide. ${ }^{2}$

Conjectura-se que esse procedimento foi utilizado por Bristow antes de 1929 ; $^{3}$ entretanto, maiores detalhes técnicos foram relatados somente em 1958 por Helfet, ${ }^{4}$ que descreveu de forma pormenorizada a técnica que ele havia aprendido em 1939 com o próprio Bristow. Em 1954, Michel Latarjet ${ }^{5}$ estabeleceu os conceitos modernos dessa cirurgia com o uso de um parafuso para fixar o processo coracoide e a possibilidade de abertura do subescapular. Patte et $\mathrm{al}^{6}$ disseminaram essa técnica na Europa continental nos anos 1980, com a utilização de dois parafusos de $4.5 \mathrm{~mm}$ para a fixação do enxerto em posição vertical.

A transferência do processo coracoide e do tendão conjunto para a borda ântero-inferior da cavidade glenoidal foi uma técnica modificada muitas vezes, mas todas as modificações respeitaram os princípios básicos de triplo bloqueio: bloqueio ósseo pelo processo coracoide, aumento da tensão da porção inferior do músculo subescapular, e bloqueio direto pelo tendão conjunto. ${ }^{7}$ Muitas dessas modificações foram bem sucedidas, e também apresentaram conhecidas complicações, sendo as mais comuns a perda da rotação lateral, osteoartrose, dor, alterações no nervo musculocutâneo e pseudoartroses. ${ }^{8}$

Essas complicações fizeram com que muitos cirurgiões abandonassem esse procedimento e preferissem a reconstrução labral, principalmente com a evolução tecnológica da artroscopia.

Recentemente, a evolução contínua dos métodos minimamente invasivos possibilitou que esse procedimento pudesse ser realizado por artroscopia de forma reprodutível com o Latarjet artroscópico. ${ }^{7}$ Por meio da visão intra-articular, o cirurgião pôde posicionar melhor o enxerto, minimizando complicações como osteoartrose e instabilidade. A inserção pela visão intra-articular garante também que a cavidade medular óssea possa estar presente nas áreas de contato, permitindo melhora da osteointegração. $7,9,10$

Entretanto, essa abordagem requeriu novos instrumentos, com aumento dos custos cirúrgicos.

Em 2009, foi publicada uma nova técnica cirúrgica artroscópica que viabilizava a transferência do enxerto do processo coracoide e do tendão conjunto pela via minimamente invasiva sem custos associados ao uso de novos materiais especiais. Nessa técnica, o enxerto é inserido na posição horizontal na borda anterior da cavidade glenoidal com o uso de materiais comuns de artroscopia associado ao uso de um parafuso maleolar e arruela, técnica conhecida mais comumente como procedimento de Bristow. ${ }^{11}$

Neste estudo, são apresentados os resultados de pelo menos de dois anos de seguimento em 43 pacientes submetidos a essa técnica para tratamento da instabilidade anterior do ombro. 


\section{Métodos}

De setembro de 2007 a dezembro de 2016,48 pacientes foram submetidos a procedimento cirúrgico para o tratamento da instabilidade anterior do ombro pela técnica de Bristow artroscópico com enxerto em posição horizontal por um mesmo cirurgião. Trata-se de estudo retrospectivo, com avaliação pré-cirúrgica considerada como referência para as avaliações.

De todos pacientes, os três primeiros não foram submetidos a escore pré-cirúrgico ou pós-cirúrgico com seguimento superior a dois anos, e os dados de outros dois pacientes foram perdidos.

Os 43 pacientes restantes preencheram os critérios de inclusão, e foram avaliados neste estudo.

\section{Critérios de Inclusão}

Os critérios de inclusão foram: pacientes com mais de 18 anos; seguimento mínimo de 2 anos; Bankart ósseo com perda maior do que $20 \%$ da glenoide (avaliado por ressonância magnética ou tomografia computadorizada pela porcentagem da perda do diâmetro da circunferência da cavidade glenoide inferior na direção de seu maior eixo de perda óssea); avulsão umeral do ligamento glenoumeral (AULGU); falha de cirurgias artroscópicas de Bankart prévias ; índice de severidade da instabilidade maior do que seis; e atletas de esporte de contato em nível competitivo com qualquer perda óssea. ${ }^{12}$

\section{Critérios de Exclusão}

Os critérios de exclusão foram: pacientes sem avaliação de referência; pacientes com perdas de dados pré-cirúrgicos ou com no mínimo dois anos de pós-cirúrgico; aqueles que não concordaram em serem avaliados; menores de idade ou pessoas de populações vulneráveis; e pacientes com outras cirurgias prévias, exceto a cirurgia artroscópica de Bankart. Escores e Mensurações

Os seguintes escores e mensurações foram usados:

O escore modificado da University of California at Los Angeles (UCLA), que foi inicialmente criado para avaliar artroplastias do ombro, mas modificações possibilitaram que ele pudesse ser usado para outras condições ortopédicas do ombro. ${ }^{13}$

O teste simples do ombro (TSO), um dos testes mais reconhecidos na avaliação funcional do ombro.

O escore de Rowe; criado para ser apenas uma avaliação pós-cirúrgica, seus resultados devem ser comparados com os pontos de corte 75 e 90, acima dos quais encontramos bons e ótimos resultados, respectivamente.

A perda de rotação lateral com braço em adução, que utilizou como referência a avaliação pré-cirúrgica, e a goniometria realizada manualmente com dados anotados em intervalos de $5^{\circ}$, sendo anotada apenas a diferença.

E a elevação, em que perdas maiores do que $10^{\circ}$ foram anotadas.

\section{Avaliações}

Na avaliação de referência, a rotação lateral e o escore da UCLA dos pacientes foram medidos. Com dois anos ou mais após a cirurgia, os pacientes foram avaliados utilizando-se as medidas da rotação lateral, elevação, e os resultados dos escores da UCLA e de Rowe, e do TSO.

As avaliações pós-cirúrgicas incluíram exames radiográficos em todos pacientes. As tomografias computadorizadas pós-cirúrgicas ficaram restritas àqueles pacientes com suspeita de complicações.

\section{Estatística}

A análise estatística foi realizada com o auxílio do software Stata 15 (StataCorp., College Station, TX, US) para Macintosh.

Os dados foram testados quanto à sua normalidade e avaliados de acordo com sua natureza estatística usando curvas bicaudais e valores de $p<0,05$. Sempre que possível, os dados foram avaliados em intenção para tratar (IPT). ${ }^{14} \mathrm{~A}$ análise interina do tamanho da amostra foi realizada verificando seu poder estatístico.

As causas de retirada de pacientes do estudo foram relatadas.

\section{Técnica Cirúrgica}

Os pacientes foram colocados em posição de cadeira de praia sob anestesia geral, e foi utilizada técnica cirúrgica padronizada com quatro portais: o portal posterior, o anterolateral, um portal logo acima do processo coracoide, e outro, anteromedial trans-subescapular; esses dois últimos portais são estabelecidos com auxílio de cateter intravenoso $2,1 \times 45 \mathrm{~mm}$, dando a segurança de seu melhor posicionamento (-Fig. 1). ${ }^{11}$

Com a ótica no portal posterior, a abertura do tendão do músculo subescapular é feita de forma ampla na direção das fibras, com uma pinça tipo Kelly forte, eletrocautério e shaver, através do portal anteromedial trans-subescapular (-Fig. 2). 0 intervalo rotador e a origem do ligamento coraoacromial na borda súpero-lateral do processo coracoide são removidos com eletrocautério e shaver. A cavidade glenoidal é cruentizada inserindo-se o shaver pelo portal anterolateral. Após isso, a broca passa pelo espaço aberto no tendão do subescapular, através do portal ântero-medial trans-subescapular, e toca a borda da cavidade glenoidal anteroinferior. Muda-se a ótica

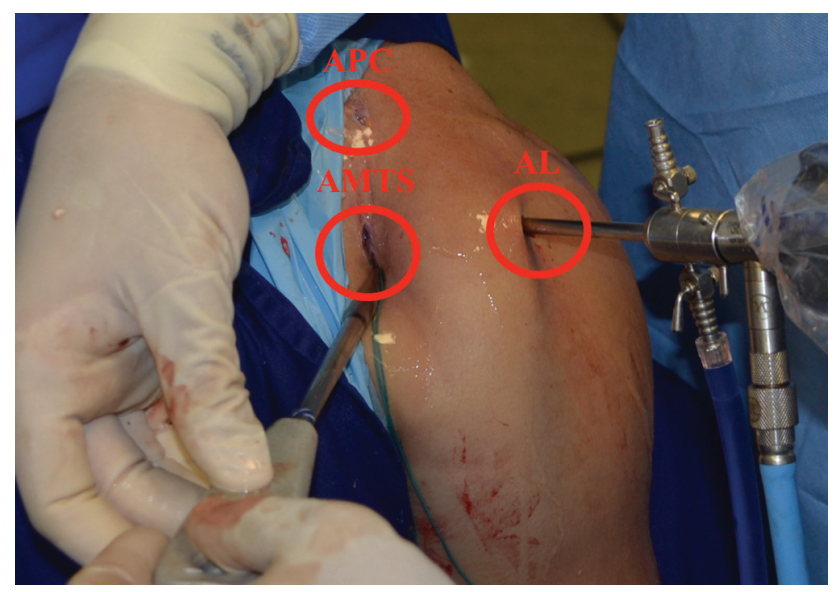

Fig. 1 Portais: APC, Acima do Processo Coracóide; AMTS, Anteromedial trans-subscapularl; AL, Anterolateral. 


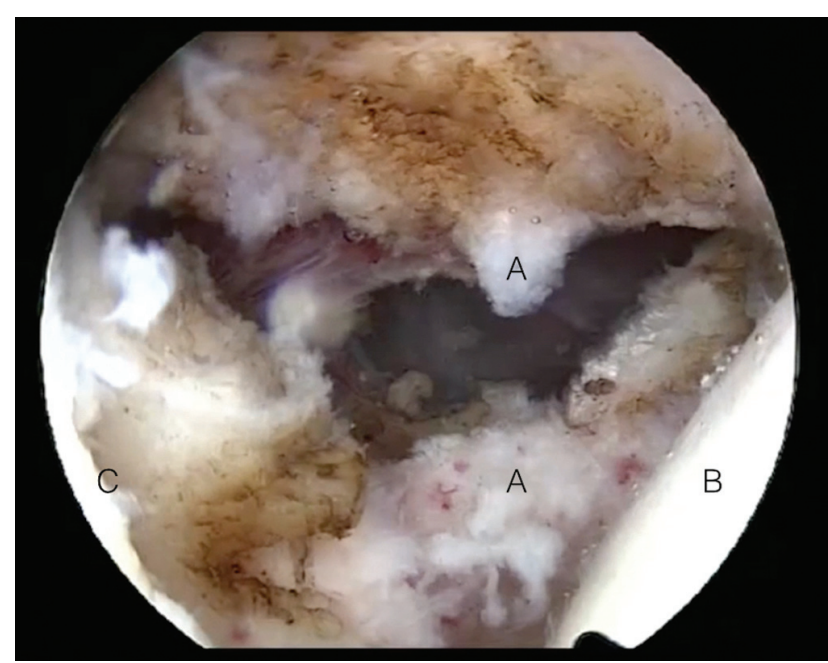

Fig. 2 Imagem artroscópica do ombro demonstrando a abertura do tendão do subescapular. A) subescapular; B) úmero; C) cavidade glenoidal.

para o portal ântero-lateral para melhor visualização, e um orifício é feito entre $5 \mathrm{~mm}$ e $6 \mathrm{~mm}$ medialmente à borda anterior da cavidade glenoidal. ${ }^{15} \mathrm{O}$ tamanho desse orifício é medido. A ótica sai do espaço intra-articular para o subdeltoide anterior, o tendão do peitoral menor é liberado com eletrocautério pelo portal acima do processo coracoide, e a osteotomia do processo coracoide é feita por esse mesmo portal com o auxílio de microsserra e osteótomos. O uso de uma pinça tipo Kocher inserida previamente no tendão conjunto pelo portal ântero-medial pode facilitar sua exteriorização (-Figs. 3 e 4). O parafuso maleolar de $3,5 \mathrm{~mm}$ a ser usado deve ter o tamanho da soma da profundidade da cavidade glenoidal e o tamanho medido do enxerto após sua exteriorização pelo portal ânteromedial. Deve-se sempre usar arruela nesse procedimento.

Coloca-se um fio de náilon multifilamentar número 5 ou um fio de alta resistência número 2 entre a arruela e a cabeça do parafuso, a fim de tracioná-lo contra a chave, evitando que o parafuso desprenda-se dela, e fixa-se o enxerto na cavidade glenoidal passando pela abertura feita no tendão do subescapular (-Figs. 5 e 6). Uma pinça tipo Kocher é inserida

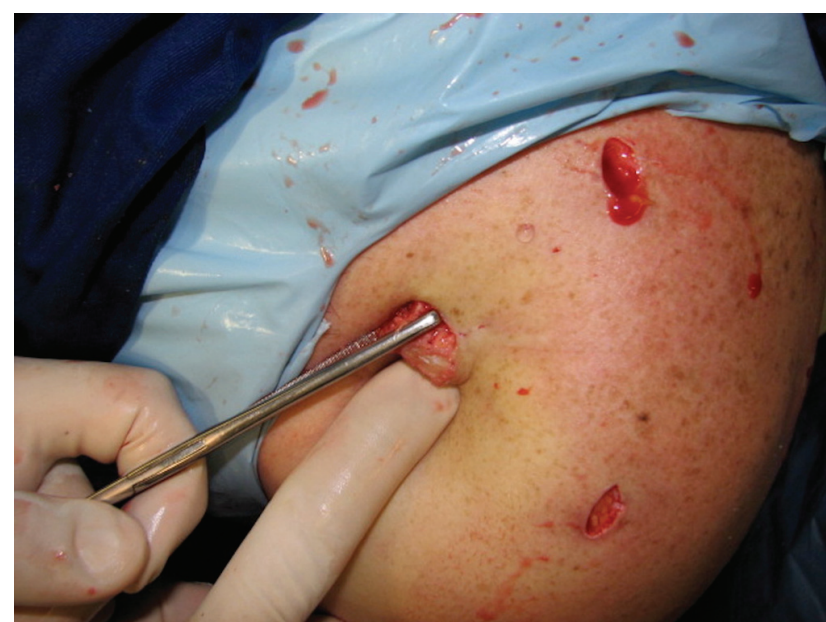

Fig. 3 Imagem do ombro esquerdo com o processo coracoide exteriorizado com o auxílio de uma pinça tipo Kocher.

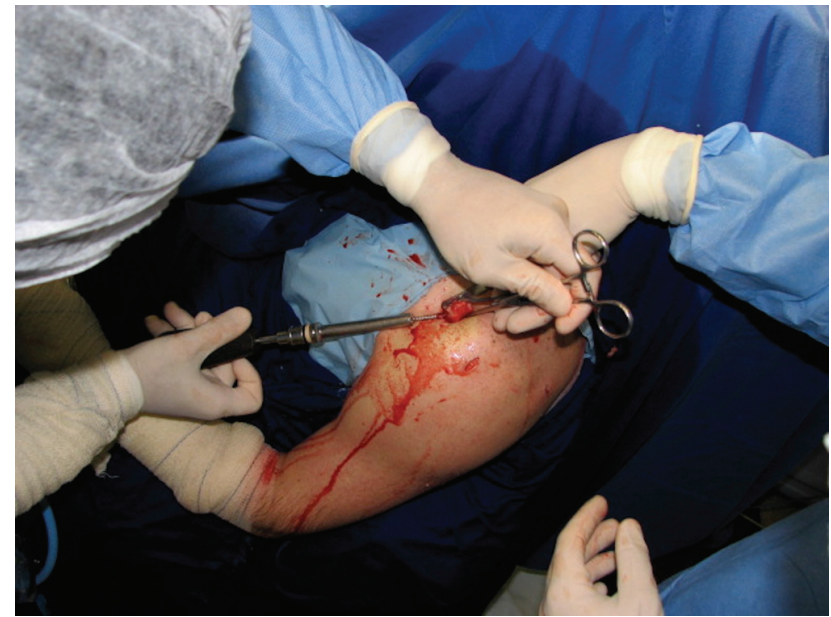

Fig. 4 Passagem do parafuso pelo enxerto do processo coracoide exteriorizado.

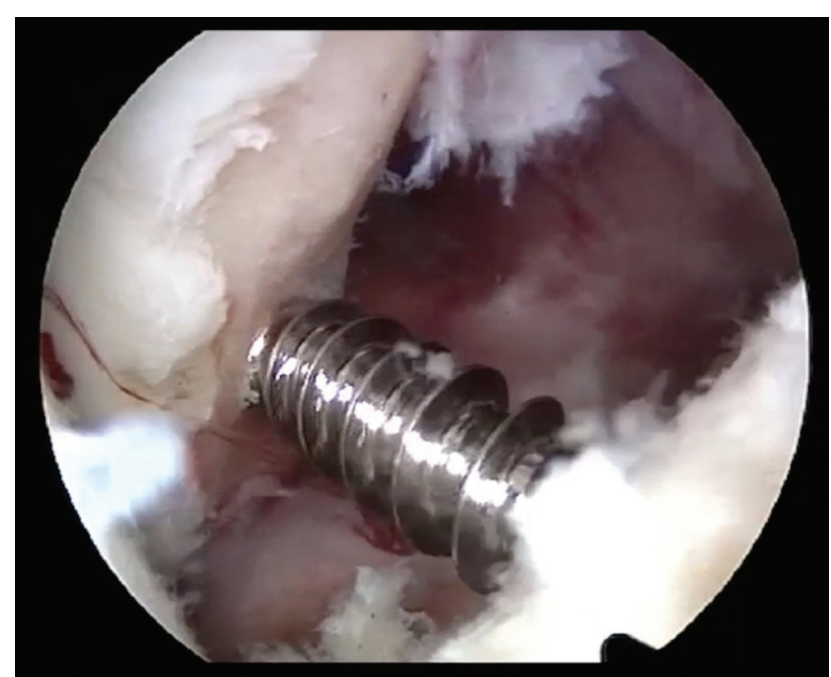

Fig. 5 Imagem artroscópica do ombro demonstrando fixação na cavidade glenoide anterior; artroscópio no portal ântero-lateral.

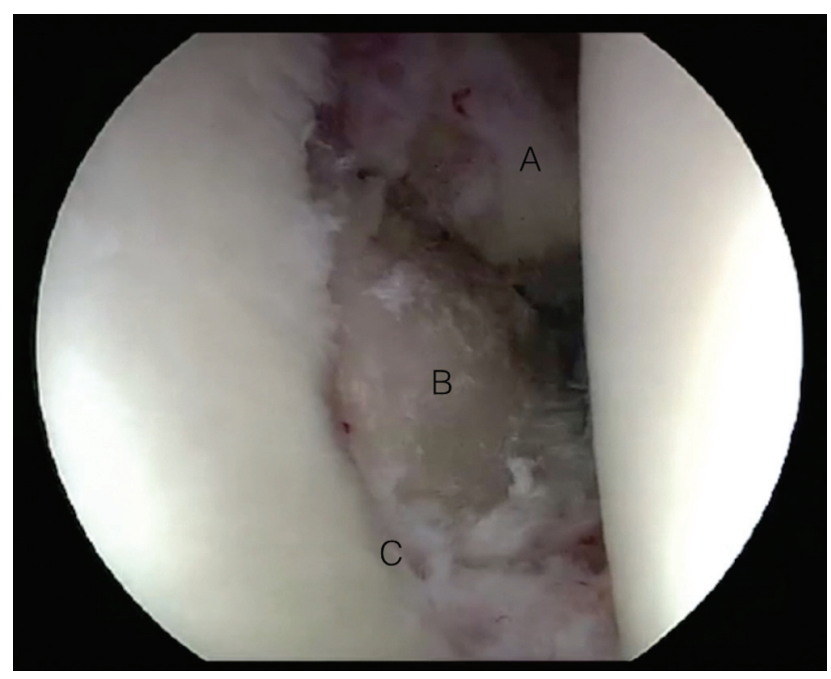

Fig. 6 Imagem artroscópica do ombro demonstrando fixação final do enxerto do processo coracoide na cavidade glenoide anterior; artroscópio no portal posterior. A) subscapular; B) coracoide inserido; C) cavidade glenoidal. 
pelo portal acima do processo coracoide, com leve compressão, para controlar a rotação do enxerto durante a fixação.

Se o enxerto apresentar degrau articular, o shaver ósseo poderá ser usado para a sua regularização.

O aumento de torque no parafuso pode não significar necessariamente que o enxerto está devidamente fixo; para testar até que ponto apertar o parafuso, deve-se usar-se a sonda para testar se a arruela está solta ou fixa.

\section{Resultados}

Dos 43 pacientes examinados na avaliação de referência, 3 não puderam ser avaliados no período pós-cirúrgico. Com os dados da avaliação de referência desses pacientes, eles foram mantidos, e seus escores, copiados da avaliação de referência para a avaliação de dois anos (IPT), a fim de evitar qualquer viés que pudesse favorecer o procedimento.

O grupo avaliado foi composto por 42 homens e 1 mulher, com média etária de 32,88 anos (18-60 anos), sendo que 15 foram submetidos ao procedimento no lado esquerdo, e 28 , no direito. O seguimento médio foi de 76 meses (129-24 meses).

As causas para realizar esses procedimentos foram: 28 pacientes apresentavam Bankart ósseo com $20 \%$ ou mais de perda óssea; 1 apresentava AULGU; 9 tinham falhas de procedimentos prévios de Bankart; e 5 tinham índice de severidade de instabilidade maior do que 6 .

A média do escore da UCLA para os 43 pacientes (IPT), e a do de Rowe, assim como a perda de rotação lateral e o TSO, estão resumidos na - Tabela $\mathbf{1}$.

Os dados resultantes são estatisticamente consistentes. ${ }^{16}$

Não houve diferenças de elevação em relação ao lado oposto maiores do que $10^{\circ}$, exceto em dois casos.

A rotação medial não foi avaliada; entretanto, 10 de 40 pacientes apresentaram desconforto, e 1, incapacidade durante os pontos extremos desse movimento (questão 11 do TSO).

\section{Complicações Intraoperatórias (43 Pacientes)}

Ocorreram duas fraturas do processo coracoide, uma incompleta, na qual o processo coracoide foi cerclado com náilon multifilamentar número 5 , e então realizado o procedimento do Bristow artroscópico. A outra fratura foi completa e multifragmentar; optou-se então por fazer uma tenodese do tendão conjunto na porção anterior da cavidade glenoidal trans-subescapular por artroscopia, com excelente resultado funcional em longo prazo. Este último paciente foi um dos três que não puderam ser avaliados no pós-cirúrgico, visto não ter sido submetido ao procedimento em estudo.

Não ocorreram lesões nervosas, paresias ou parestesias em nenhum dos casos.

\section{Complicações Pós-cirúrgicas (40 Pacientes)}

Houve três pseudoartroses sem repercussões clínicas.

Ocorreu uma torção de parafuso de $8,84^{\circ}$ na direção medial do plano axial sem repercussões clínicas, e o enxerto se consolidou mesmo após torção.

Ocorreram quatro osteólises, três sem repercussão clínica, e em uma foi necessária a retirada do parafuso. Osteoartrose estava presente em três pacientes, dois com quadro moderado e um inicial, de acordo com a classificação de Samilson e Prieto $;{ }^{17}$ dois desses pacientes já apresentavam osteoartrose moderada antes do procedimento cirúrgico.

Um paciente teve infecção possivelmente de origem hematogênica seis meses após o procedimento. Impacto anterior estava presente em dois pacientes, incluindo um com associação de osteólise do enxerto, e foi necessária a retirada do material de síntese em ambos. Não houve subluxações ou reluxações após o procedimento.

Lesões concomitantes foram tratadas em quatro pacientes: três lesões do labrum superior, de anterior a posterior (superior labral anterior and posterior, SLAP), e uma lesão do manguito.

Entre os 43 pacientes operados, houve um total de 12 complicações, 8 das quais não apresentaram qualquer repercussão clínica. As complicações com repercussão foram a infecção seis meses após a cirurgia de possível origem hematogênica, a fratura do coracoide que fez com que o paciente precisasse mudar o procedimento no intraoperatório, e impacto anterior em dois pacientes, nos quais foi necessária a retirada de material de síntese mais de seis meses após a cirurgia. Desses quatro pacientes com complicações, todos apresentaram boa resposta aos tratamentos instituídos, com melhora de seus quadros clínicos, exceto o paciente com a infecção, que apresentou os piores resultados funcionais desta série.

A análise intermediária da amostra mostrou tamanho adequado, considerando-se significância de 0,001 e poder estatístico de $99 \%$. ${ }^{18,19}$

\section{Discussão}

Recentemente, modificações em procedimentos de transferência do processo coracoide têm sido relatadas, possibilitando que esse procedimento seja realizado por artroscopia. ${ }^{7,9,10}$

Tabela 1 Comparação dos valores do escore da UCLA, de Rowe, do teste simples do ombro, e da perda de rotação lateral

\begin{tabular}{|l|l|l|l|l|}
\hline & $\begin{array}{l}\text { Pré-cirúrgico ( } \pm \text { desvio padrão ou padrão } \\
\text { de bons/ótimos resultados) }\end{array}$ & $\begin{array}{l}\text { > 2 anos pós-cirurgia } \\
\text { ( } \pm \text { desvio padrão) }\end{array}$ & Valor de $\boldsymbol{p}$ & $\boldsymbol{n}$ \\
\hline UCLA & $25,56 \pm 0,50$ & $33,23 \pm 0,44$ & $<0,0001$ & 43 \\
\hline Rowe & 75 (bons resultados) & $94,25 \pm 1,52$ & $<0,0001$ & 40 \\
\hline Rowe & 90 (ótimos resultados) & $94,25 \pm 1,52$ & 0,0082 & 40 \\
\hline TSO & - & $11,35 \pm 0,21$ & - & 40 \\
\hline Perda de rotação lateral & - & $10,37 \pm 1,36$ & - & 40 \\
\hline
\end{tabular}

Abreviaturas: TSO, teste simples do ombro; UCLA, University of California at Los Angeles. 
As vantagens do método artroscópico são: melhor visualização do sítio de inserção do enxerto; possibilidade de correção de degraus articulares com visão articular; visualização intra-articular do enxerto com certeza de sua correta localização; capacidade de testar a rotação lateral com visão articular; possibilidade de tratamento de outras lesões de forma concomitante; redução de aderências; e melhora estética. ${ }^{1,7}$ As desvantagens são: aumento de custos relacionados à artroscopia; curva de aprendizado demorada; e necessidade de treinamento específico. ${ }^{7}$

A fratura do processo coracoide foi a pior complicação nesse procedimento (três casos). Edwards e Walch ${ }^{20}$ sugerem a técnica dos dois dedos, na qual o torque do parafuso é realizado apenas com o polegar e o indicador, evitando torque excessivo do parafuso sobre o enxerto. A visualização e o teste da arruela, no qual se passa a sonda sobre a arruela para se ter certeza de que ela está fixa, também pode aumentar a segurança do procedimento, visto que em alguns casos o aumento do torque sentido na chave pode não ser a compressão do enxerto, mas a fixação na cortical contralateral. Quanto ao tamanho do parafuso, os de $3,5 \mathrm{~mm}$ não canulados e com arruela parecem ser os mais adequados. Os cuidados mencionados anteriormente eliminaram as fraturas do processo coracoide nesta série. Em casos de fratura em que cerclagem não seja possível ou viável, o autor sugere tenodese do tendão conjunto na porção anterior da cavidade glenoidal com âncoras.

Estudos em cadáveres não têm encontrado diferenças biomecânicas significativas entre a tenodese do tendão conjunto e a osteossíntese do processo coracoide com tendão conjunto, sugerindo que não há necessidade do bloqueio ósseo a fim de se atingir a estabilidade do ombro em procedimentos cirúrgicos. ${ }^{21,22}$ Há menção de que a estabilidade do ombro possa estar mais ligada aos mecanismos passivos e ativos de partes moles de difícil mensuração. 23

Na verdade, nem no procedimento de Bristow nem no de Latarjet, conforme descritos originalmente, há quantidade de osso suficiente para produzir o efeito de bloqueio ósseo. No procedimento de Bristow descrito por Helfet, o processo coracoide é suturado na porção anterior da glenoide, e no de Latarjet, o processo coracoide é osteotomizado antes do início da inserção do peitoral menor, preservando completamente a inserção desse músculo, com enxerto de tamanho muito reduzido., ${ }^{4}$

$\mathrm{O}$ efeito de bloqueio ósseo certamente adicionou estabilidade às variações modernas dos procedimentos de Bristow e de Latarjet; entretanto, talvez esse benefício seja apenas sentido em pacientes que apresentem lesões de Hill-Sachs em uma região a uma distância da inserção do manguito que seja maior ou igual ao diâmetro original da cavidade glenoidal menos a perda óssea vezes $0,83 .^{24}$

Há uma discussão na literatura quanto ao diâmetro do parafuso para transferências do coracoide. Walch e Boileau ${ }^{25}$ usam o de $4,5 \mathrm{~mm}$; Burkhart et al ${ }^{26}$ e Di Giacomo et $\mathrm{al}^{27}$ usam o de $3.75 \mathrm{~mm}$ canulado; e Lafosse et $\mathrm{al}^{7}$ usam o de $3.5 \mathrm{~mm}$. Todos os autores citados usam enxerto na posição vertical. $\mathrm{Na}$ técnica apresentada no presente estudo, o diâmetro do parafuso dependerá das características físicas do paciente, mas há clara predileção pelo de $3,5 \mathrm{~mm}$ não canulado acompanhado de arruela.
Walsh e Boileau ${ }^{25}$ recomendam não utilizar arruelas; isso ocorre pelo uso de parafusos grandes e pela proximidade da arruela quando o enxerto é deixado na posição vertical. Nesse procedimento com um parafuso menor, há benefícios mecânicos no uso da arruela, que é recomendado por duas razões: para a distribuição da carga, e por ela ser a opção de teste quando há dúvida se o aperto do parafuso vem do torque contra a cortical contralateral ou de fato da compressão do enxerto.

No procedimento de Bristow artroscópico, a osteólise do enxerto foi encontrada em apenas 3 casos (nenhum nos últimos 15 pacientes). Como a osteólise pode ser associada às forças de compressão impostas ao enxerto, talvez os métodos de fixação utilizando a técnica de dois dedos e o teste da arruela tenham sido fundamentais para a obtenção desses resultados. ${ }^{27}$ No entanto, há um possível viés, visto que as avaliações pós-cirúrgicas foram realizadas somente por radiografia, deixando os exames de tomografia apenas para os casos em que se desconfiava de possíveis complicações.

Exérese do parafuso foi necessária em um caso de osteólise acompanhada de impacto. ${ }^{28}$

Mesmo havendo apenas 1 retirada de material de síntese, 11 dos 40 pacientes responderam "não" ou que apresentavam desconforto na questão 11 do TSO. Dessa forma, certo impacto pode ser subestimado nesta série. $O$ autor aceita que pode haver algum impacto sem ou com pouca repercussão clínica em até $27,5 \%$ dos pacientes. A retirada do parafuso foi necessária em $2 \mathrm{~s}$ dos 11 pacientes com impacto. $O$ impacto pode ser associado ao ângulo do enxerto, que deve ser perpendicular ao traço da fratura, e preferencialmente não maior do que o ângulo da continuidade da circunferência da cavidade glenoidal. A visualização direta e dinâmica pelo espaço intra-articular permite ao cirurgião ter maior controle do posicionamento do enxerto, evitando os degraus articulares. $O$ autor sugere que o tamanho $\mathrm{e}$ a obliquidade do enxerto são fatores determinantes tanto no impacto quanto na profundidade glenoidal efetiva. ${ }^{29}$

Neste estudo, a taxa de reoperação foi de $5 \%$, taxa similar à de relatos anteriores para o procedimento aberto. ${ }^{8}$

A osteoartrose já estava presente em dois pacientes antes do procedimento, e apenas um caso apresentou sinais radiográficos novos. Essa baixa taxa de osteoartrose pode estar associada à visão direta com correções intraoperatórias que evitam o degrau articular.

A ausência do degrau articular diminui a carga sobre o enxerto por não haver ponto de estresse mecânico, não ocasionando mudanças importantes na pressão sobre o quadrante póstero-superior da glenoide. ${ }^{10}$ Tem-se demonstrado que não só a lateralização do enxerto, como também a sua medialização está associada a complicações pós-cirúrgicas. Acima de $5 \mathrm{~mm}$ de medialização, há associação com maiores índices de reincidência da instabilidade do ombro. ${ }^{30}$ A melhor avaliação intraarticular da região de fixação pela via artroscópica pode ser um fator positivo para se evitar essa complicação.

Até o momento, por não haver recorrências da instabilidade e pelos seguimentos de qualidade de vida demostrarem resultados estatisticamente significantes, é possível afirmar a eficácia da técnica.

Neste estudo, foram observadas três pseudoartroses, mas nenhuma teve repercussões clínicas, assim como é relatado 
na literatura. A quantidade das pseudoartroses pode estar sendo subestimada pelo fato de o segmento ter sido em sua maioria realizado com radiografias convencionais, o que pode dificultar esse diagnóstico.

Sugere-se que a utilização de apenas um parafuso seja o suficiente para a fixação do processo coracoide. Hovelius et $\mathrm{l}^{30}$ relataram 11 reluxações para 319 ombros, 3\%, no procedimento de Bristow aberto. Desses pacientes, $13 \%$ apresentavam pseudoartrose, mas a união fibrosa foi suficiente para evitar reluxações. Houve maior associação das recorrências com a medialização do enxerto do que com as pseudoartroses. ${ }^{30}$

A ausência de recorrência da luxação neste estudo pode estar associada ao melhor posicionamento do enxerto, que melhorou o contato da área escarificada da cavidade glenoide anterior com a do osso esponjoso do enxerto na posição horizontal; entretanto, a amostra ainda é baixa para sugerir tais conclusões. Taxas baixas, próximas a $1 \%$ de reluxação, em mais de 2.346 procedimentos cirúrgicos, são citadoas na literatura para esse procedimento quando realizado por via aberta, e os resultados deste trabalho sugerem essa mesma tendência de bons resultados para o procedimento artroscópico. ${ }^{31}$

Taxas de reluxação menores não estão associadas ao uso de dois parafusos por técnicas similares. ${ }^{26,32}$

A alteração pós-cirúrgica do nervo musculocutâneo não foi observada; na literatura, sua incidência é de $0,7 \%{ }^{10}$ Mesmo sem comprometimento neurológico, séries maiores são necessárias para que se tire qualquer conclusão com respeito à superioridade da segurança neurológica do procedimento em questão.

O procedimento em estudo tem apresentado tempo de internação igual ao de outros procedimentos artroscópicos similares, com vantagem significativa do ponto de vista econômico, devido ao uso exclusivo de materiais básicos de artroscopia, um parafuso de pequenos fragmentos, e uma arruela.

\section{Conclusão}

O procedimento de Bristow artroscópico mostrou eficácia extremamente significativa no tratamento da instabilidade anterior do ombro; entretanto, não é um procedimento livre de complicações, alcançando um índice de complicações com repercussão clínica de $9 \%$ nesta série.

Conflitos de Interesse

$\mathrm{O}$ autor é consultor da Zimmer-Biomet.

\section{Referências}

1 Zacchilli MA, Owens BD. Epidemiology of shoulder dislocations presenting to emergency departments in the United States. J Bone Joint Surg Am 2010;92(03):542-549

2 Boileau P, Villalba M, Héry JY, et al. Risk factors for recurrence of shoulder instability after arthroscopic Bankart repair. J Bone Joint Surg Am 2006;88(08):1755-1763

3 Page CM, Bristow WR. The treatment of fractures and dislocations in general practice. 3rd ed. Oxford: Oxford Medical Publications; 1929

4 Helfet AJ. Coracoid transplantation for recurring dislocation of the shoulder. J Bone Joint Surg Br 1958;40-B(02):198-202
5 Latarjet M. A propos du traitement des luxations récidivantes de l'épaule. Lyon Chir 1954;49(08):994-997

6 Patte D, Bernageau J, Rodineau J, et al. [Unstable painful shoulders (author's transl)]. Rev Chir Orthop Repar Appar Mot 1980;66(03): 157-165

7 Lafosse L, Lejeune E, Bouchard A, et al. The arthroscopic Latarjet procedure for the treatment of anterior shoulder instability. Arthroscopy 2007;23(11):1242.e1-1242.e5

8 Griesser MJ, Harris JD, McCoy BW, et al. Complications and reoperations after Bristow-Latarjet shoulder stabilization: a systematic review. J Shoulder Elbow Surg 2013;22(02):286-292

9 Boileau P, Bicknell RT, El Fegoun AB, et al. Arthroscopic Bristow procedure for anterior instability in shoulders with a stretched or deficient capsule: the "belt-and-suspenders" operative technique and preliminary results. Arthroscopy 2007;23(06):593-601

10 Nourissat G, Nedellec G, O'Sullivan NA, et al. Mini-open arthroscopically assisted Bristow-Latarjet procedure for the treatment of patients with anterior shoulder instability: a cadaver study. Arthroscopy 2006;22(10):1113-1118

11 Garcia JC, Garcia JPM, Mattos CA, et al. Arthroscopic BristowLatarjet-Like procedure: Surgical technique. Tech Shoulder Elbow Surg 2009;10:94-98

12 Balg F, Boileau P. The instability severity index score. A simple preoperative score to select patients for arthroscopic or open shoulder stabilisation. J Bone Joint Surg Br 2007;89(11):1470-1477

13 Romeo AA, Bach BR Jr, O'Halloran KL. Scoring systems for shoulder conditions. Am J Sports Med 1996;24(04):472-476

14 Streiner D, Geddes J. Intention to treat analysis in clinical trials when there are missing data. Evid Based Ment Health 2001;4(03): 70-71

15 Young AA, Baba M, Neyton L, et al. Coracoid graft dimensions after harvesting for the open Latarjet procedure. J Shoulder Elbow Surg 2013;22(04):485-488

16 Pocock SJ. When to stop a clinical trial. BMJ 1992;305 (6847):235-240

17 Samilson RL, Prieto V. Dislocation arthropathy of the shoulder. J Bone Joint Surg Am 1983;65(04):456-460

18 Harmonised Tripartite Guideline ICH. Statistical principles for clinical trials (E9). 1998. Available at: http://www.ich.org/fileadmin/Public_Web_Site/ICH_Products/Guidelines/Efficacy/E9/ Step4/E9_Guideline.pdf

19 Peacock JL, Peacock PJ. Sample size for comparative studies: means. In: Peacock JL, Peacock PJ, editors. Oxford handbook of medical statistics. 3rd ed. Oxford: Oxford University Press; 2011

20 Edwards TB, Walch G. The Latarjet procedure for recurrent anterior shoulder instability: rationale and technique. Oper Tech Sports Med 2002;10(01):25-32

21 Thomas PR, Parks BG, Douoguih WA. Anterior shoulder instability with Bristow procedure versus conjoined tendon transfer alone in a simple soft-tissue model. Arthroscopy 2010;26(09):1189-1194

22 Yamamoto N, Muraki T, An KN, et al. The stabilizing mechanism of the Latarjet procedure: a cadaveric study. J Bone Joint Surg Am 2013;95(15):1390-1397

23 Panchal AP, Osbahr DC, Douoguih W, et al. Conjoined tendon transfer vs modified Bristow in a glenoid bone loss model: A biomechanical study. Orthop J Sports Med 2013;1(4 Suppl): 2325967113S00012

24 Yamamoto N, Itoi E, Abe H, et al. Contact between the glenoid and the humeral head in abduction, external rotation, and horizontal extension: a new concept of glenoid track. J Shoulder Elbow Surg 2007;16(05):649-656

25 Walsh G, Boileau P. Latarjet-Bristow procedure for recurrent anterior instability. Tech Shoulder Elbow Surg 2000;1:256-261

26 Burkhart SS, De Beer JF, Barth JR, et al. Results of modified Latarjet reconstruction in patients with anteroinferior instability and significant bone loss. Arthroscopy 2007;23(10):1033-1041

27 Giacomo GD, Costantini A, de Gasperis N, et al. Coracoid bone graft osteolysis after Latarjet procedure: A comparison study 
between two screws standard technique vs mini-plate fixation. Int J Shoulder Surg 2013;7(01):1-6

28 Ghodadra N, Gupta A, Romeo AA, et al. Normalization of glenohumeral articular contact pressures after Latarjet or iliac crest bone-grafting. J Bone Joint Surg Am 2010;92(06):1478-1489

29 Lippitt SB, Vanderhooft JE, Harris SL, et al. Glenohumeral stability from concavity-compression: A quantitative analysis. J Shoulder Elbow Surg 1993;2(01):27-35

30 Hovelius L, Sandström B, Olofsson A, et al. The effect of capsular repair, bone block healing, and position on the results of the
Bristow-Latarjet procedure (study III): long-term follow-up in 319 shoulders. J Shoulder Elbow Surg 2012;21(05):647-660

31 Garcia JCJr, do Amaral FM, Belchior RJ, et al. Comparative Systematic Review of Fixation Methods of the Coracoid and Conjoined Tendon in the Anterior Glenoid to Treat Anterior Shoulder Instability. Orthop J Sports Med 2019;7(01):2325967118820539

32 Collin P, Rochcongar P, Thomazeau H. [Treatment of chronic anterior shoulder instability using a coracoid bone block (Latarjet procedure): 74 cases]. Rev Chir Orthop Repar Appar Mot 2007;93 (02):126-132 